\title{
Role of Hsp70 Subfamily, Ssa, in Protein Folding in Yeast Cells, Seen in Luciferase-Transformed ssa Mutants
}

\author{
Keiko Unno, ${ }^{*}$ Takahiro KISHIdo, Maho Hosaka, and Shoji OKada \\ Department of Radiobiochemistry, School of Pharmaceutical Sciences, University of Shizuoka, 52-1, Yada, Shizuoka \\ 422, Japan. Received June 19, 1997; accepted August 19, 1997
}

\begin{abstract}
To investigate the role of constitutive hsp70 in protein folding and to probe the supplementation by other hsps in this folding, yeast cells expressing reduced constitutive hsp70 proteins, ssalssa2, were transformed with a plasmid expressing a bacterial luciferase protein. With several independent clone cells of transformants, the levels of luciferase activity and some hsps, such as hsp104, hsp90, hsp70 and hsp26, were examined. The luciferase activity was significantly lower in ssaIssa2 transformants than in the wild type (wt) cell transformed with the same plasmid. Among several clone cells of ssa1ssa2, the cells with higher luciferase activities exhibited higher amounts of Ssa4 which is known to be expressed instead of lacking Ssa1 and Ssa2. The luciferase activity closely correlated with the amount of Ssa proteins, more than with the amount of other hsps. It is suggested that constitutional Ssa "chaperones" are needed for the folding of proteins and, in cells lacking Ssa1 and Ssa2, the increased Ssa4 is thought to partly compensate for their role in the folding of luciferase in vivo.
\end{abstract}

Key words hsp70; luciferase; protein folding; molecular chaperone; yeàst

Molecular chaperones (stress proteins, heat shock proteins (hsps)) have been shown to have essential roles in the functional processes of proteins, such as folding, assembly, translocation, reactivation and proteolysis. ${ }^{1-5)}$ They perform complex and ingenious collaborations in cells. Moreover, hsp families contain some members whose function and structure are similar. Actually, such collaborations and compensation might be significant for the maintenance of a living system under unfavorable conditions.

Although constitutive hsp 70 proteins have been thought to play an important role in protein folding, the lack of both constitutive hsp70s of Ssal and Ssa2 (ssalssa2) is not fatal in Saccharomyces cerevisiae ${ }^{6-8)}$ Some other hsps overexpressed in the mutant cells ${ }^{9,10)}$ might compensate for the role played by Ssa1 and Ssa2. To elucidate the role of the constitutive Ssa chaperones in protein folding and to probe the supplementary role of other hsps, ssalssa2 cells expressing luciferase were used in this study. It has been shown in studies of luciferase proteins that de novo protein folding is mediated by GroE chaperones ${ }^{11)}$ and restoration of denatured protein is needed for hsp70 in E. coli. ${ }^{12)}$ In yeast, it has been found that hsp104, but not hsp70, mediates resolubilization of heat-inactivated luciferase. ${ }^{13)}$

Interestingly, the basal level of luciferase activity is significantly low in ssalssa2 cells. It is suggested that constitutional members of Ssa are necessary for the folding of proteins, at least luciferase protein. Luciferase activity is rapidly increased by heat treatment, suggesting that heat-inducible hsps also have a role in the folding of luciferase. By measuring luciferase activity and the level of some hsps, such as hsp104, hsp90, hsp70 and hsp26, in several independent cell clones of ssalssa2, we found that ssalssa 2 cells with a higher luciferase activity had a higher level of Ssa4. This result suggests that Ssa4 partly assumes the role of protein folding in cells lacking Ssal and Ssa2.

* To whom correspondence should be addressed.

\section{MATERIALS AND METHODS}

Yeast Cells Carrying Luciferase Plasmid Saccharomyces cerevisiae cells of the wild-type (strain A1591) and the hsp70 mutant (strain A1630) were kindly gifted by Dr. S. Lindquist of The University of Chicago. The latter cell is the double mutant lacking the constitutive hsp70 proteins of Ssa1 and Ssa2 (ssalssa2), ${ }^{6}$ and expressing a bacterial luciferase protein ${ }^{14)}$ from the constitutive glyceraldehyde 3-phosphate dehydrogenase promoter (pLux(URA)). ${ }^{13}$ The wild-type (wt) cell similarly expresses luciferase protein. These cells were grown at $25^{\circ} \mathrm{C}$ to the mid-log phase in minimal dextrose medium supplemented with nutrients excluding uracil (URA $(-)$ ). To transform ssalssa2 cells to express $S S A 1$, they were transfected with a plasmid expressing SSA1 on YEp351 which was kindly donated by Dr. E. A. Craig of The University of Wisconsin.

Detection of Hsps Yeast cells $\left(2 \times 10^{7}\right.$ cells $)$ were homogenized in $0.2 \mathrm{ml}$ ethanol containing $2 \mathrm{~mm}$ phenylmethylsulfonyl fluoride (PMSF) with $200 \mathrm{mg}$ glass beads. After removing ethanol, the homogenate was resuspended in sodium dodecyl sulfate (SDS) sample buffer containing $0.05 \mathrm{~m}$ Tris- $\mathrm{HCl}$ buffer ( $\mathrm{pH}$ 6.8), $1 \%$ 2-mercaptethanol, $1 \%$ SDS, and $10 \%$ glycerol. The cell lysate was separated by electrophoresis on $10 \%$ or $7.5 \%$ SDS-polyacrylamide gel and transferred to a polyvinylidene fluoride (PVDF) transfer membrane (Immobilon-P, Millipore). The membranes were processed with antiserum as described below. Complexes of antibody and hsp were visualized by using horseradish-peroxidase-conjugated second antibody and the ECL ${ }^{\mathrm{TM}}$ detection system (Amersham).

The expression of hsp70 was detected with a specific monoclonal antibody (MAb 7.10) and an antibody against Ssa (anti-SSA). The former recognizes all members of Ssa hsp70, and the latter recognizes Ssa1/2, but not Ssa3/4. Hsp26, hsp90, hsp104, Ssb and Kar2 were detected with yeast specific antibodies. Ssb and Kar2 are members of Saccharomyces hsp70, and located in the cytosol (ribosome) and endoplasmic reticulum, respectively. The 
antibodies of MAb 7.10, anti-hsp26, anti-hsp90 and anti-hspl04 were kindly gifted by Dr. S. Lindquist. The antibodies of Ssa and Ssb were kindly provided by Dr. E. Craig. Anti-Kar2 antibody was provided by Dr. M.D. Rose of The Princeton University.

Amounts and Activities of Luciferase Total cellular proteins were extracted from the cells using glass-beads and separated by SDS-polyacrylamide gel electrophoresis (SDS-PAGE) as described above. These proteins were transferred to a PVDF membrane and reacted with luciferase-specific antiserum, which was provided by Dr. A. Escher of University of Alberta. Immune complexes were detected with the ECL Western blot detection kit. Luciferase activity was assayed in vivo by the addition of $10 \mu \mathrm{l} n$-decylaldehyde (Sigma) to $1 \mathrm{ml}$ or $200 \mu \mathrm{l}$ of the $\log$ phase culture. Light emission was measured immediately with a Tropix luminometer or Lumiphotometer (Labo Science). The activity was measured in triplicate. To examine the effects of heat treatment on luciferase activity, cells were heated at $37 \mathrm{C}$ for $30 \mathrm{~min}$ followed by incubation at $25 \mathrm{C}$ for $1-2 \mathrm{~h}$.

Protein Synthesis in ssalssa2 The cells in the mid-log phase were pre-incubated with medium excluding methionine at $25 \mathrm{C}$ for $1 \mathrm{~h}$ and labeled with a $\left[{ }^{35} \mathrm{~S}\right]$ amino acid mixture consisting of ${ }^{35} \mathrm{~S}$-labeled L-methionine and L-cysteine (Pro-mix ${ }^{\mathrm{TM}}$ L- $\left[{ }^{35} \mathrm{~S}\right]$, Amersham) $(37 \mathrm{kBq} / 5 \times$

(a)

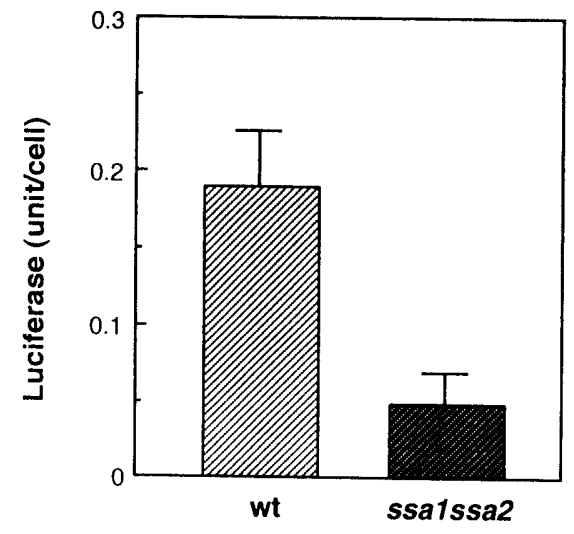

(b)

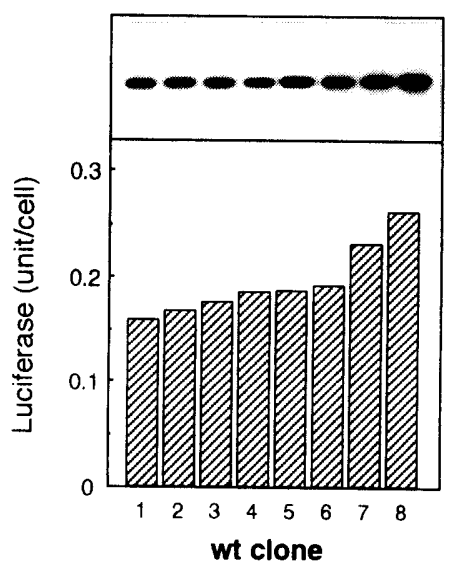

(c)

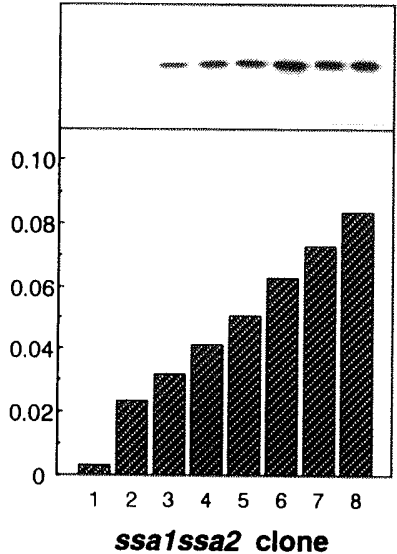

Fig. 1. Activity and Amount of Luciferase in wt and ssalssaz Cells

(a): Mean $\pm S . I)$. $(n=10)$ of luciferase activity of wt and ssalswaz transformant cells. (b) and (c): The amount (upper panels) and activity (lower panels) of luciferase of cach clone (no. 1 8) of wt (b) and ssatsere (c) cells.
$10^{6}$ cells $/ \mathrm{ml}$ ) for $30 \mathrm{~min}$. The radioactivity incorporated into the protein fraction was compared between the sistasisat and wt cells.

\section{RESULTS}

Activity and Amount of Luciferase in ssalssa2 The activity of luciferase was tested in several different clones which formed independent colonies on a URA(-) agar plate. In wt cells, the average activity of 10 independent colonies was $0.19 \pm 0.04$ unit/cell. In ssalssa2 cells, the activity varied from 0.003 to 0.084 unit/cell, and the average activity was $0.048 \pm 0.020 \mathrm{unit} / \mathrm{cell}$ (Fig. 1a).

To investigate the reason for the low activity of luciferase in the ssalssa2 cells, the synthesis of the proteins was analyzed in the transformant cells with a level of luciferase activity that was close to the mean. Incorporation of 1. $-\left[{ }^{35} \mathrm{~S}\right]$ amino acids into the proteins in the ssalssat 2 cells $(41000 \pm 882 \mathrm{dpm} / \mu \mathrm{g}$ protein) was not lower than that in the wt cells $(38440 \pm 408 \mathrm{dpm} / \mu \mathrm{g}$ protein). The amount and activity of the enzyme in the wt and ssalssa 2 cells were determined and arranged in increasing order of the activity as shown in Figs. $1 b$ and c, respectively. As seen in these figures, the enzyme activity per cell was almost proportional to the amount of the enzyme. This result suggests

Table 1. Increased Relative Activity of Luciferase Following HeatTreatment

$\begin{array}{lcc}\text { Treatment } & \begin{array}{c}\text { Relative activity of luciferase } \\ \text { (ratio to control) }\end{array} \\ & \text { wt } & \text { ssalssat } \\ \text { Control }(25 \mathrm{C}) & 1.00 & 1.00 \\ 37 \text { C } 30 \mathrm{~min} & 0.97 & 1.14 \\ 37 \text { ( } 30 \mathrm{~min}+25 \mathrm{C}, 1 \mathrm{~h} & 2.31 & 4.24 \\ 37 \mathrm{C} 30 \mathrm{~min}+25 \mathrm{C}, 2 \mathrm{~h} & 3.80 & 2.86\end{array}$

(a) MAb 7.10

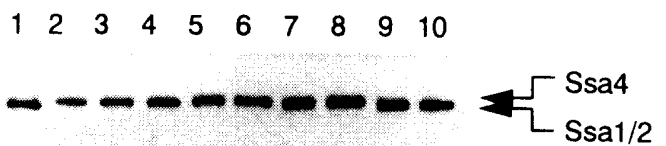

(b) anti-SSA

$\begin{array}{llllllllll}1 & 2 & 3 & 4 & 5 & 6 & 7 & 8 & 9 & 10\end{array}$

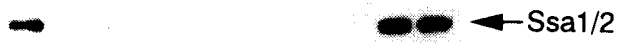

Fig. 2. Difference in the Amount of Ssia Chaperones between wt and ssalssar Cells

(a): Hsp70, detected with anti-hsp70 antibody, MAb 7.10), in wt cells (lanes 1, 9 and 10$)$ and ssalssuz cells (lanes 28 ). All members of Ssa react with the antibody. (b): Hsp70, detected with anti-Ssal 2 antibody. anti-SSA, in wl and ssalswa cells. The cells in each lane correspond to those in (a). 


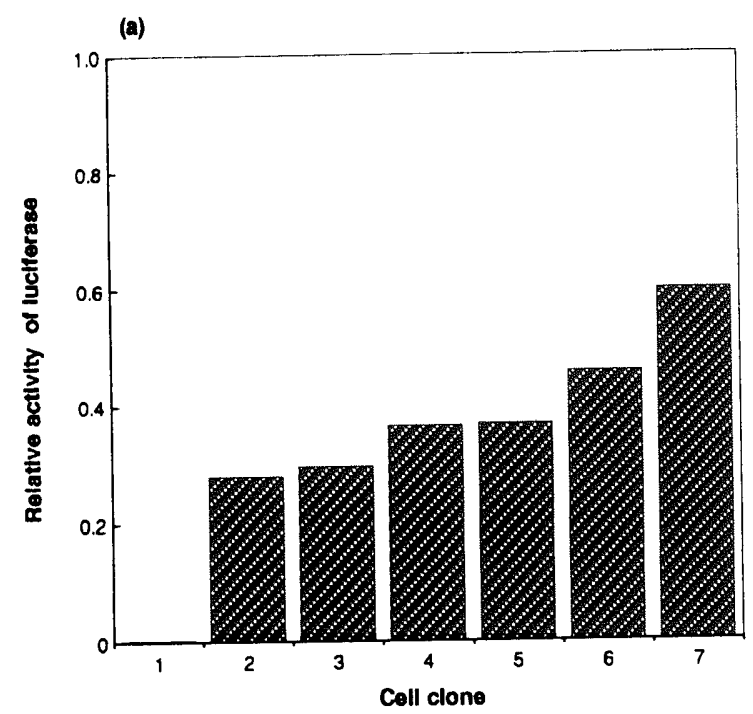

(b)
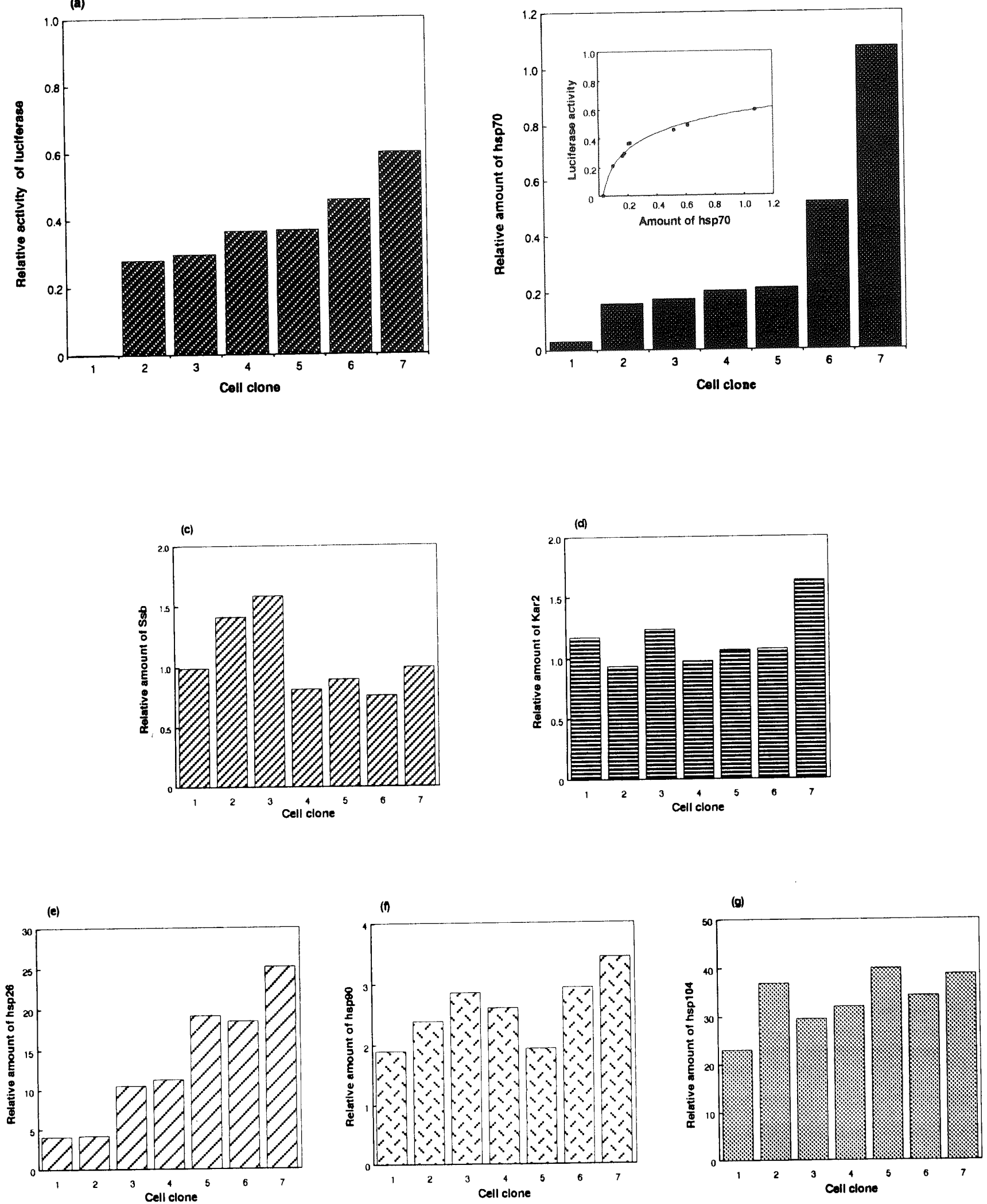

Fig. 3. Relationship between Luciferase Activity and the Amount of Ssa Chaperones or Other hsps in ssalssa2 Cell Clones (no. 1-7)

(a) (a): Luciferase activity of each clone relative to wt (mean of s samples). (b). Amount of Ssa cha (g): Amounts of Ssb (c). Kar2 (d). hsp26 (e), hsp90 (f) and hspl04 (g). figure shows the relationship between luciferase activity and the at a the in wi cells.

that the unfolded form of luciferase synthesized is unstable in vivo.

Expression of Hsps in the ssalssa2 Cells After heat treatment at $37 \mathrm{C}$, the activity of luciferase was greater than the control during the following incubation at $25^{\circ} \mathrm{C}$ for $1-2 \mathrm{~h}$ in both ssalssa2 and wt cells (Table 1). The heat treatment induced some hsps, such as hsp 104, hsp70 and hsp26, without changing luciferase activity. The 
(b)

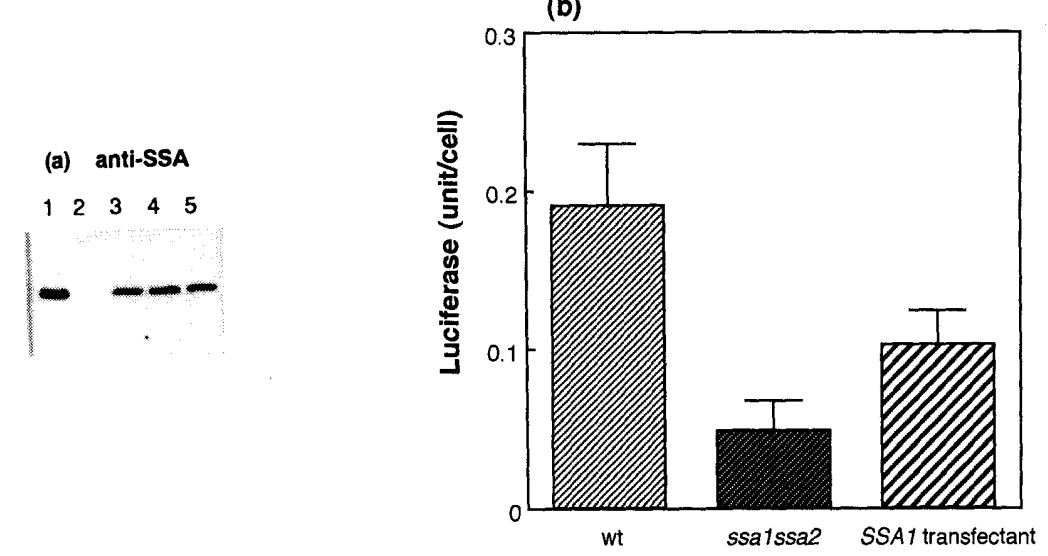

Fig. 4. Effect of Transfection of $S S A 1$ on Luciferase Activity

(a): Ssal/2 in wt (lane 1), original ssalssa2 (lane 2) and each clone of SSA1 transfectant (lanes 3-5) cells were detected with anti-SSA antibody. (b): Luciferase activity of wt, ssalssa 2 and $S S A 1$ transfectant cells.

increased luciferase activity was likely caused by some induced hsp. The amount of hsps in the ssalssa2 cells of each clone of different luciferase activity was assayed by Western immunoblot analysis. Each member of the hsp70 family proteins was detected using the antibodies, MAb 7.10 and anti-SSA. The amount of hsp 70 in ssalssa 2 clone cells, arranged in increasing order of luciferase activity (Fig. 2a, lanes 2-8), was compared with that in wt cells whose activity was close to the mean (lanes 1,9 and 10). It was found that the cells with higher luciferase activity exhibited a higher amount of hsp70 and the mobility of hsp70 in ssalssa2 cells was a little slower than that of wt cells. The bands in ssalssa2 were not detected with anti-SSA (Fig. 2b, lanes 2-8). These results indicated that Ssa4, a heat-inducible hsp70 with a slightly slower mobility, was overexpressed in the ssalssa2 cells under the non-heated conditions (Figs. 2a and b), as previously described by Craig et al. ${ }^{7,10,15)}$

The cells with a higher luciferase activity exhibited a higher amount of Ssa4 (Figs. 3a and b), showing a correlation between the two parameters (Fig. $3 b$ inset). The amount of other hsp70 family proteins, Kar2 and Ssb, in ssalssa2 was similar to or a little higher than that in wt (Figs. 3c and d). On the other hand, other hsps, hsp26, hsp90 and hsp104, were significantly overexpressed in the ssalssa2 cells (Figs. 3e-g). The amount of these hsps might not explain the decreased luciferase activity.

Effect of Transfection of SSA1 Plasmid on ssalssa2 Cells To confirm the effect of the lack of SSA1 and SSA2, ssalssa2 cells were transfected with an SSA1 plasmid. Transfectant cells expressing SSA1 (Fig. 4a, lanes 3-5) exhibited a higher luciferase activity than that of the original ssalssa2 cells (Fig. 4b). This result suggests that the expression of Ssal is important for luciferase activity.

\section{DISCUSSION}

The role of constitutive hsp 70 in protein folding in yeast cells was investigated using a luciferase-transformed mutant. In the ssalssa2 cells, the amount and activity of luciferase were significantly lower than those in the wt cells (Fig. 1a). This phenomenon of low expression was not found in the hsp104 mutant cell transformed with luciferase. ${ }^{13)}$ On the other hand, the rate of cellular protein synthesis was not lower in the ssalssa2 than in the wt cell. It has been reported that tranlational elongation is slower than the folding process ${ }^{16)}$ and, thus, polypeptides are folded on the ribosome. ${ }^{17)}$ In cells lacking a significant chaperone, the amount of folded protein might depend on the amount of chaperones. In ssalssaz cells, the activity of luciferase corresponded to the amount (Figs. 1b and c), suggesting that unfolded luciferase is unstable in vivo and the lack of constitutive hsp70, Ssa1 and Ssa2, is responsible for the low activity of luciferase. Furthermore, the different activity of luciferase in each of the clone cells and the increased activity following heat treatment (Table 1) suggest a compensatory role for hsp members, other than Ssal and Ssa2, in protein folding.

We found that ssalssa2 cells with a higher luciferase activity exhibited a higher amount of Ssa4 (Figs. 3a and b). There was a positive correlation between luciferase activity and the amount of $\mathrm{Ssa} 4$ (Fig. 3b inset). It is thought that Ssa4 partly, but not completely, compensated for the lack of Ssal and Ssa2 in the folding of luciferase. On the other hand, no relationship was found between luciferase activity and the level of Ssb, Kar2, hsp26, hsp90 and hsp104 (Fig. 3c-g). Although the level of hsp26 was also positively correlated, the cells with low luciferase activity exhibited a much higher amount of hsp26 than those of $\mathrm{wt}$, suggesting that the alteration of hsp26 expression did not cause the low activity of luciferase.

The ssalssa2 cells transfected $S S A 1$ plasmid exhibited a higher luciferase activity than those of the original cells (Fig. 4). The folding of TRP3 protein, a yeast gene product, has been reported to be partially dependent on Ssa chaperones for its activity. ${ }^{16)}$ Although possible involvement of other hsps could not be excluded, the present data support a significant involvement of constitutive Ssa chaperones in the protein folding in vivo as well as in vitro. ${ }^{18)}$ Our study also suggests that there is a supplementary role for Ssa4, one of the chaperones induced in the cells lacking Ssa1 and Ssa2. However, as the folding of most of the other endogenous proteins was scarcely affected by the lack of Ssal and Ssa2, Ssa and some other chaperones might be involved in the protein folding. Protein folding in vivo is thought to be carried 
out by complex and ingenious collaborations and compensation by molecular chaperones.

Acknowledgements The authors gratefully acknowledge Professor S. Lindquist of The University of Chicago for her very helpful advice and generous donation of yeast cells and antibodies. We also thank Dr. Y. Kimura of The Tokyo Metropolitan Institute of Medical Science for her useful advice.

\section{REFERENCES}

1) Craig E. A., Weissman J. S., Horwich A. L., Cell, 78, 365-372 (1994).

2) Geogopoulos C., Welch W., Annu. Rev. Cell Biol., 9, 601-634 (1993).

3) Hendrick J. P., Hartl F.-U., Annu. Rev. Biochem., 62, 349-384 (1993).

4) Hendrick J. P., Hartl F.-U., FASEB J., 9, 1559-1569 (1995).

5) Parsell D. A., Lindquist S., Annu. Rev. Genet., 27, 437--496 (1993).
6) Craig E. A., Jacobsen K., Cell, 38, 841--849 (1984).

7) Craig E. A., Jacobsen K., Mol. Cell. Biol., 5, 3517-3524 (1985).

8) Werner-Washburne M., Stone D. E., Craig E. A., Mol. Cell. Biol., 7, 2568-2577 (1987).

9) Nelson R. J., Heschl M. F. P., Craig E. A., Genetics, 131, 277-285 (1992).

10) Halladay J. T., Craig E. A., Mol. Cell. Biol., 15, 4890-4897 (1995).

11) Escher A., Szalay A. A., Mol. Gen. Genet., 238, 65-73 (1993).

12) Schröder H., Langer T., Hartl F.-U., Bukau B., EMBO J., 12 , 4137-4144 (1993).

13) Parsell D. A., Kowal A. S., Singer M. A., Lindquist S., Nature (London), 372, 475-478 (1994).

14) Escher A., O'Kane D. J., Lee J., Szalay A. A., Proc. Natl. Acad. Sci. U.S.A., 86, 6528-6532 (1989).

15) Boorstein W. R., Craig E. A., J. Biol. Chem., 265, 18912-18921 (1990).

16) Crombie T., Boyle J. P., Cogging J. R., Brown A. J. P., Eur. J. Biochem., 226, 657-664 (1994).

17) Kolb V. A., Makeyev E. V., Spirin A. S., EMBO J., 13, 3631--3637 (1994).

18) Levy E. J., McCarty J., Bukau B., Chirico W. J., FEBS Lett., 368, $435-440(1995)$ 